\title{
Displasia fibrosa: relato de três casos Filbrous displasia:
}

\author{
Adriana L. Alves ${ }^{1}$, Fernando Canavarros ${ }^{2}$, \\ Daniela S. A. Vilela ${ }^{2}$, Lídio Granato ${ }^{3}$, \\ José D. Próspero
}

Palavras-chave: displasia fibrosa, recidiva, diagnóstico diferencial, fibroma ossificante.

Key words: fibrous dysplasia, recurrence, differential diagnostic, ossifying fibroma.

\section{Resumo / Summary}

\begin{abstract}
$\mathbf{A}$
displasia fibrosa é lesão pseudo-neoplásica de etiologia desconhecida, caráter benigno e recidivante, caracterizada pelo desenvolvimento de tecido fibroso e traves osteóides que substituem gradualmente o osso normal. O principal diagnóstico diferencial da forma monostótica, no envolvimento dos ossos da cabeça e pescoço, é o fibroma ossificante, que alguns consideram variante desta entidade. Esse trabalho tem como objetivo revisar as principais características clínicas, radiológicas e histopatológicas que auxiliam no diagnóstico diferencial da displasia fibrosa com o fibroma ossificante e discutir o comportamento recidivante da displasia fibrosa, essencial ao planejamento cirúrgico. Relatamos três casos provenientes do ambulatório de Otorrinolaringologia da Santa Casa de Misericórdia de São Paulo, avaliados quanto às queixas, exame físico, achados de imagem e tratamentos. Nos três casos o diagnóstico foi confirmado pelo exame anatomopatológico. Todos apresentaram recidiva após a ressecção cirúrgica, diagnosticada entre o primeiro e o oitavo ano de seguimento. Devido ao comportamento clínico semelhante da displasia fibrosa e do fibroma ossificante, o exame anatomopatológico é essencial para o diagnóstico diferencial. O acompanhamento clínico permanente desses pacientes é necessário para que as possíveis recidivas sejam diagnosticadas precocemente.
\end{abstract}

\begin{abstract}
F dysplasia is a pseudo-neoplastic lesion, ethiology unknown, benign and recurrent, which normal bone is replaced by fibrous tissue and lamelar bone trabeculae. The main differential diagnosis of the monostotic form on head and neck bones is Ossifying Fibroma which some consider another form of the same entity. The purpose of this study is to make a review of the main clinical, radiological and histopathological findings that contributes to the differential diagnosis. The recurrent behavior of Fibrous Dysplasia is essential to its surgical planning and it was also analyzed on this study. We have reported three cases followed at the ENT department of Irmandade da Santa Casa de Misericórdia de São Paulo evaluated based on the patients symptoms, physical exam, imaging findings and treatment. In all three cases, the diagnosis was confirmed based on histopathological findings. All of them had recurrences after surgical removal, diagnosed between first and eighth year of follow up. Due to Fibrous Dysplasia and Ossifying Fibroma similar clinical courses, the histopathological findings are essential to their differential diagnosis. The long term follow up of this pacients is necessary in order to make an early diagnosis of recurrences.
\end{abstract}

\footnotetext{
${ }^{1}$ Pós-Graduanda da Faculdade de Medicina da Santa Casa de Misericórdia de São Paulo, na área de Otorrinolaringologia.

${ }^{2}$ Médicos residentes do Departamento de Otorrinolaringologia da Santa Casa de Misericórdia de São Paulo

${ }^{3}$ Diretor do Departamento de Otorrinolaringologia da Santa Casa de Misericórdia de São Paulo

${ }^{4}$ Livre Docente de Anatomia Patológica da Faculdade de Ciências Médicas da Santa Casa de Misericórdia de São Paulo.
}

Endereço para correspondência: Departamento de Otorrinolaringologia da Santa Casa de São Paulo

Rua Dr Cesário Mota Júnior, 112 - Pavilhão Conde de Lara - 4º andar - Telefone (0xx11) 3226-7235 - Fax: (0xx11) 2228405

Trabalho realizado no Departamento de Otorrinolaringologia da Santa Casa de Misericórdia de São Paulo, apresentado sob a forma de tema livre no $35^{\circ}$ Congresso Brasileiro de Otorrinolaringologia realizado no período de 16 a 20 de outubro de 2000, na cidade de Natal.

Artigo recebido em 6 de setembro de 2001. Artigo aceito em 20 de novembro de 2001 


\section{INTRODUÇÃO}

A displasia fibrosa monostótica é lesão pseudoneoplásica benigna de etiologia ainda desconhecida. Representa cerca de $2,5 \%$ de todos os tumores ósseos. Por tratar-se de defeito congênito da modelagem óssea, manifesta-se mais freqüentemente em crianças e adolescentes, tendo crescimento lento e tendência a se estabilizar após a puberdade, junto com o esqueleto. Apresenta alta taxa de recidiva, com valores em torno de $37 \%$ nos adultos ${ }^{1}$. À microscopia é característica a presença de tecido fibroso em meio ao qual existem traves osteóides irregulares que lembram caracteres chineses.

Devido à predileção pelos ossos da face e crânio, geralmente causando deformidade, é doença de particular interesse pelo otorrinolaringologista. As formas poliostóticas nesta região determinam leontíase óssea.

Serão descritos três casos de portadores de displasia fibrosa, com diagnóstico confirmado pelo exame anatomopatológico, recidivantes.

\section{APRESENTAÇÃO DOS CASOS}

\section{Caso 1}

Masculino, nove anos, pardo, com queixa de cefaléia frontal latejante há dois anos, com piora matutina, às vezes acompanhada de vômitos. Referia também diminuição da acuidade visual à esquerda. Ao exame físico o paciente apresentava proptose do globo ocular à esquerda.

A tomografia de seios da face mostrou espessamento ósseo irregular em seio frontal à esquerda, seios etmoidais à esquerda e seio esfenoidal, com nervo óptico preservado (Figura 1).

O paciente foi submetido à craniotomia frontal

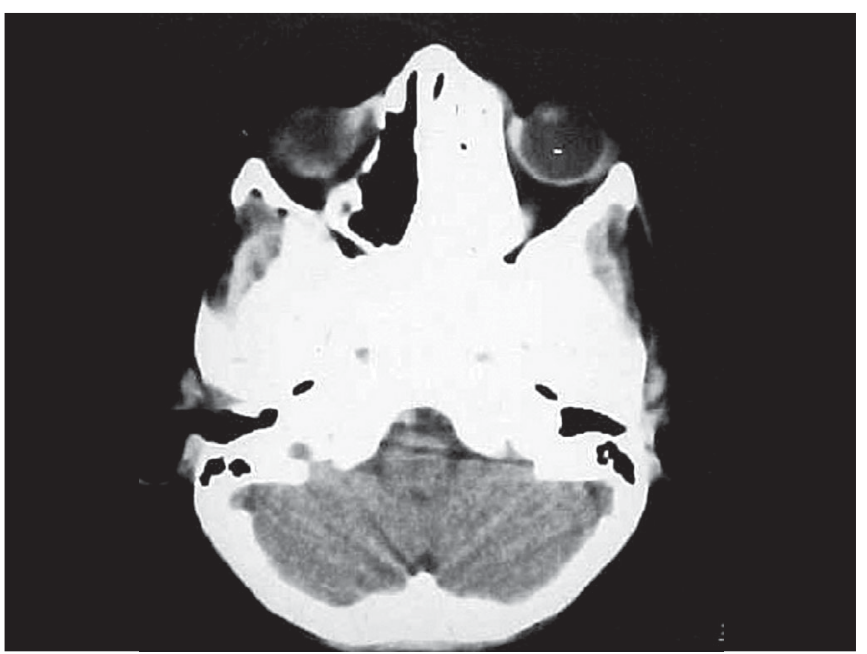

Figura 1. Corte axial de tomografia computadorizada de base de crânio mostrando osso displásico ocupando fossa nasal esquerda, seios etmoidais e esfenoidal. esquerda para exérese de tumor orbitário, cujo exame anatomopatológico revelou ser displasia fibrosa.

Após oito anos o paciente voltou a apresentar cefaléia. A tomografia computadorizada de seios da face mostrou recidiva do tumor ósseo, com expansão para seios etmoidais, esfenoidal e frontal à esquerda.

O paciente foi submetido à frontoetmoidectomia e esfenoidectomia à esquerda para exérese do tumor.

O exame anatomopatológico confirmou o diagnóstico anterior. O paciente está no segundo mês de pós-operatório, assintomático e sem sinais de recidiva.

\section{Caso 2}

Masculino, oito anos, branco, com queixa de sangramento nasal e episódios repetidos de conjuntivite purulenta.

Ao exame físico apresentava hipertelorismo, drenagem purulenta pelo saco lacrimal à esquerda; nariz com base alargada e fossa nasal esquerda preenchida por tumor vinhoso.

A tomografia de seios da face revelou a presença de massa expansiva em fossa nasal esquerda e etmóide, homogênea, com aspecto de vidro fosco (Figura 2).

O paciente foi submetido à cirurgia para retirada do tumor por via transpalatina.

O exame anatomopatológico diagnosticou displasia fibrosa.

Dois anos depois o paciente voltou a apresentar queixas de obstrução nasal, associada a episódios de sangramento nasal, deslocamento do globo ocular para a esquerda e diminuição da acuidade visual.

Ao exame clínico apresentava hipertelorismo, proptose do olho esquerdo; abaulamento da pirâmide nasal à esquerda, deslocamento da arcada dentária superior para

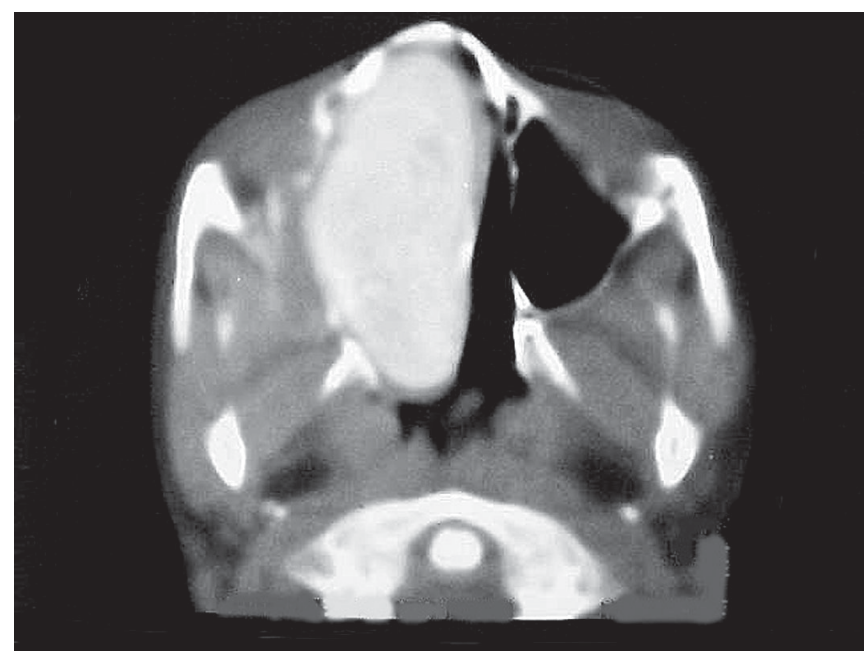

Figura 2. Corte axial de tomografia computadorizada de seios da face mostrando tumor com densidade homogênea ocupando a cavidade nasal à esquerda, com aspecto de vidro fosco. 
a direita e fossa nasal esquerda preenchida por tumor vinhoso. Realizada tomografia computadorizada de seios da face que mostrou processo expansivo ocupando a fossa nasal esquerda, parede medial do seio maxilar esquerdo, seios etmoidais, parte do esfenóide, lâmina pterigoidea medial à esquerda e parede medial e superior da órbita esquerda, reduzindo a cavidade orbitária e deslocando o globo ocular para a esquerda.

Foi realizada nova intervenção cirúrgica com abordagem tipo Degloving sendo encontrada grande quantidade de osso displásico entre a órbita esquerda e o seio maxilar, e na região do etmóide.

O exame anatomopatológico confirmou o diagnóstico anterior.

O paciente está no $18^{\circ}$ mês de pós-operatório, sem queixas e com melhora da deformidade facial.

\section{Caso 3}

Feminino, 14 anos, branca, com queixa de obstrução nasal à esquerda há um ano, acompanhada de cefaléia periorbitária e em região maxilar à esquerda.

Ao exame apresentava tumor endurecido, recoberto de mucosa lisa e rósea, doloroso ao toque, ocupando todo o teto da fossa nasal esquerda.

A tomografia computadorizada de seios da face mostrou a presença de uma massa expansiva na região da fossa nasal esquerda, todo o seio etmoidal e esfenóide, com densidade óssea (Figura 3).

O paciente foi submetido à cirurgia para exérese do tumor, visualizando-se a invasão dos seios etmoidais, esfenoidal à esquerda e fossa nasal esquerda, se estendendo até a rinofaringe.

O exame anatomopatológico diagnosticou displasia fibrosa.

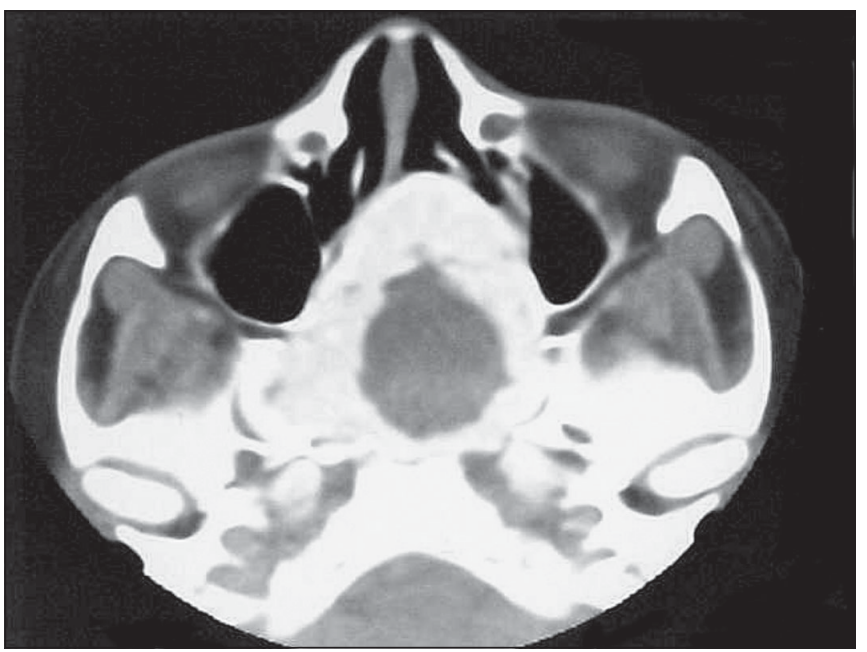

Figura 3. Corte axial de tomografia computadorizada de seios da face mostrando tumor ocupando fossas nasais, deslocando o septo para a direita, e seios esfenoidais, com densidade óssea ao redor e conteúdo cístico.
No $12^{\circ}$ mês de pós-operatório o paciente voltou a apresentar queixa de obstrução nasal à direita. A tomografia computadorizada mostrou espessamento e esclerose das paredes dos seios esfenoidais, lâminas papiráceas e vômer, com invasão do SNC, envolvendo o dorso da sela túrcica e das clinóides. Foi submetido a nova cirurgia para exérese do tumor via Degloving. O exame anatomopatológico confirmou o diagnóstico prévio de displasia fibrosa. O paciente se encontra no $10^{\circ}$ mês de pós-operatório, assintomático e com exame físico normal.

\section{DISCUSSÃO}

A displasia fibrosa é doença pouco comum, congênita, benigna, caracterizada por defeito de modelagem óssea, com substituição gradual do osso normal por fibrose com traves osteóides irregularmente mineralizadas. Alguns acreditam ser uma anomalia congênita do desenvolvimento do tecido mesenquimal ${ }^{1}$.

Representa 2,5\% dos tumores ósseos e 7,5\% dos tumores ósseos benignos ${ }^{1}$.

Cerca de $70 \%$ dos casos se manifestam na primeira década de vida, de crescimento lento, inicialmente assintomáticos, se estabilizam após a puberdade, junto com o esqueleto ${ }^{2}$. É mais freqüente no sexo feminino, recidivante em cerca de $37 \%$ dos casos nos adultos ${ }^{1}$.

A displasia fibrosa é classificada em dois tipos: monostótica, quando ocorre em um osso único ou ossos contíguos; poliostótica, quando em múltiplos ossos. A forma poliostótica, associada à presença de manchas cutâneas hiperpigmentadas e à puberdade precoce, no sexo feminino, corresponde à Síndrome de Albright ${ }^{3}$.

O envolvimento da cabeça e pescoço é comum, em cerca de 10 a 30\% das formas monostóticas e 50 a 100\% das formas poliostóticas ${ }^{1,2}$. Nesta região há divergências quanto à sua localização preferencial. Alguns acreditam ser o maxilar, seguido dos ossos frontal e etmóide ${ }^{1}$. Leed e Seaman, em 1962, identificaram o osso frontal e o esfenoidal como os

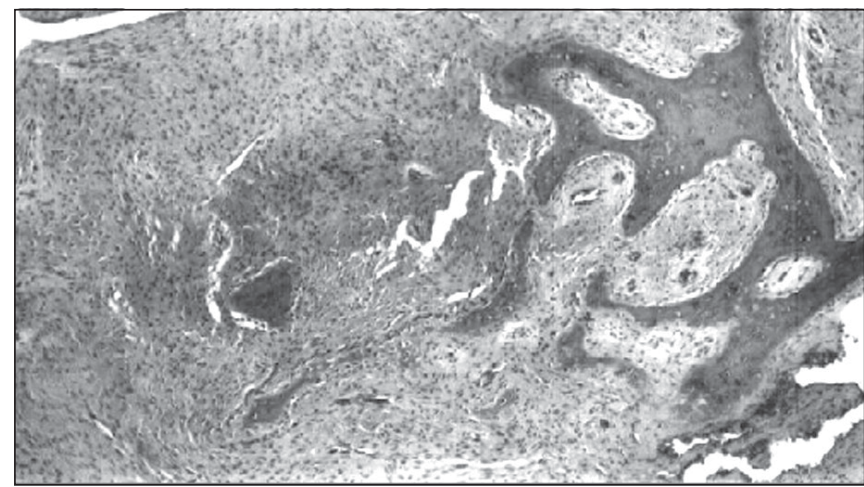

Figura 4. Corte histológico mostrando processo displásico caracterizado por proliferação fibrosa, com traves ósseas neoformadas irregulares em tamanho e forma, com variável mineralização, assumindo aspecto de caracteres chineses. 
mais comumente envolvidos ${ }^{4}$. Em nossos casos foram mais freqüentemente envolvidos o etmóide, esfenóide, frontal e maxilar.

A transformação maligna ocorre em menos de 1\% dos casos, e pós-radioterapia essa taxa pode atingir 44\% dos casos $^{1}$.

A displasia fibrosa geralmente é assintomática devido ao lento crescimento, e às vezes se manifesta com cefaléia e dor.

Os sinais e sintomas se relacionam ao seu crescimento, sendo comuns as deformidades crânio-faciais, proptose e outras complicações oculares como ceratite secundária à exposição, restrição de movimentação ocular resultando em diplopia, epífora, blefaroptose e perda do campo visual. O envolvimento do seio esfenoidal pode comprometer o canal óptico 5 . Em apenas um dos nossos casos ocorreu comprometimento ocular por compressão extrínseca.

Radiologicamente são descritas três formas de displasia fibrosa no crânio ${ }^{1}$ :

- Forma compacta: é encontrada em 50\% dos casos. O osso é progressivamente substituído de modo a ficar homogêneo, com imagem comparável a vidro fosco. Essas lesões são encontradas principalmente na base do crânio, corpo do esfenóide, teto da órbita e asa menor do esfenóide.

- Forma lítica: encontrada mais freqüentemente na calota craniana e ossos faciais. A tomografia computadorizada mostra lesão irregular, radiolúcida, circundada por halo com alta densidade.

- Forma mista: caracterizada pela presença de áreas radiopacas alternadas com áreas radiolúcidas à tomografia computadorizada. Esta foi a forma observada em nossos três casos.

Ao exame anatomopatológico, duas formas podem ser distingüidas macroscopicamente ${ }^{1}$ :

- Forma compacta: caracterizada pela presença de tecido osteóide que progressivamente se ossifica e assume aspecto de osso maduro.

- Forma cística: apresenta uma ou mais cavidades circundadas pelas alterações acima descritas.

Microscopicamente, a aparência dessas duas formas é única. Há proliferação de tecido fibroso com ossificação progressiva e destruição do osso afetado. O trabeculado ósseo é imaturo, sendo irregular no seu tamanho, formato e distribuição, com ausência da estrutura lamelar interna característica do tecido ósseo normal As traves osteóides dispõem-se de modo irregular, circundadas por osteoblastos, em arranjo que lembram caracteres chineses (Figura 4). A patogenia, segundo alguns, é a metaplasia do tecido fibroso.

O diagnóstico diferencial da displasia fibrosa se faz com a hiperostose, o osteoma, osteocondroma, cordoma, meningioma hiperostótico, granuloma reparador de células gigantes, tumor marrom do hiperparatiroidismo e o fibroma ossificante. Podem ser diferenciados pelos métodos de imagem, mas quando falhos a diferenciação é feita pelo exame anatomopatológico.

O fibroma ossificante é o principal diagnóstico diferencial da forma monostótica da displasia fibrosa, pela semelhança clínica, radiológica e anatomopatológica.

$\mathrm{Na}$ cabeça e pescoço é encontrado tipicamente na mandíbula e maxila, porém ocasionalmente pode ser observado nos seios paranasais, órbita e calota craniana.

Este processo caracteriza-se por ossificação em faixas que se anastomosam, entremeadas por proliferação fibrosa. Persiste a dúvida quanto à natureza deste processo que, segundo alguns, seria uma variante da displasia fibrosa.

Geralmente é mais agressivo que a displasia fibrosa, mas freqüentemente são indistingüíveis clinicamente ${ }^{2}$. O fibroma ossificante, ao exame radiográfico, apresenta margem definida bem evidente à tomografia computadorizada, o que facilitaria a sua diferenciação da displasia fibrosa ${ }^{6}$. Alguns autores discordam ao achar que quase todas as lesões fibroósseas benignas, incluindo a displasia fibrosa e o fibroma ossificante, mostram margens bem definidas à tomografia, impossibilitando a diferenciação.

Geralmente é heterogêneo, podendo ser uniloculado ou multiloculado. Tem crescimento excêntrico, mas geralmente não causa destruição óssea adjacente.

A ressonância nuclear magnética não ajuda na diferenciação, pois ambos apresentam sinais de baixa intensidade tanto em T1 quanto em T2, sendo heterogêneos com contraste.

O exame anatomopatológico é utilizado para o diagnóstico diferencial dessas lesões. Geralmente o fibroma ossificante tem uma cápsula óssea, que não existe na displasia fibrosa ${ }^{6}$.

As trabéculas osteóides são geralmente maduras, com alguns osteoblastos em suas bordas, e são importantes para o diagnóstico diferencial das trabéculas ósseas da displasia fibrosa, geralmente imaturas ${ }^{6}$.

Esses achados histológicos são controversos, já que em alguns casos a displasia fibrosa originada em ossos crâniofaciais tende a ser mais diferenciada $a^{6}$. O diferencial entre estas entidades é difícil pela falta de critérios diagnósticos bem definidos.

O tratamento tanto da displasia fibrosa como do fibroma ossificante é cirúrgico, objetivando sua remoção completa, pela possibilidade de recidiva e por não serem radiossensíveis. A malignização é rara em ambos.

O fibroma ossificante, apesar do crescimento lento, pode ter localmente comportamento mais agressivo. A displasia fibrosa apresenta comportamento benigno e a cirurgia radical nem sempre é justificada. A remoção pode ser dificultada pela ausência de delimitação entre o tumor e o tecido ósseo normal, sendo muitas vezes econômica na 
tentativa de evitar mutilações. A recidiva da displasia fibrosa é mais freqüente do que o fibroma ossificante e ocorreu em nossos três casos.

\section{COMENTÁRIOS FINAIS}

A displasia fibrosa é doença que acomete indivíduos jovens. Tem comportamento benigno, com crescimento geralmente lento e assintomático, mas, de acordo com sua localização, pode invadir e causar compressão de estruturas nobres na base do crânio e órbita. Essas características não devem ser esquecidas durante o planejamento cirúrgico. Por ser "tumor" sem limites precisos e recidivante, é importante que se remova o máximo de tecido possível, entretanto a cirurgia deve ser econômica para não causar mutilações e déficits funcionais. A radioterapia deve ser evitada, pelos riscos de malignização da doença e por ser ineficiente.

$\mathrm{Na}$ cabeça e pescoço, o diagnóstico diferencial da forma monostótica deve ser feito com o fibroma ossificante. Devido aos comportamentos clínico e radiológico semelhantes, o exame anatomopatológico é essencial para a diferenciação. O seguimento permanente dos pacientes é imprescindível a fim de que possíveis recidivas sejam diagnosticadas precocemente.

\section{REFERÊNCIAS BIBLIOGRÁFICAS}

1. Jan M, Dweik A, Destrieux C, Djebbari Y. Fronto-orbital sphenoidal fibrous dysplasia. Neurosurgery 1994;34(3):544-547.

2. Bailey BJ. Head and Neck Surgery-Otolaryngology. $2^{\text {nd }} \cdot$ ed. vol. 1; Philadelphia: Lippincott-Raven Publishers, 1998.

3. Paparella MM, Shumrick DA, Gluckman JL, Meyerhoff WL Otolaryngology. $3^{\text {rd }}$. ed. vol 2. Philadelphia: W.B. Sauders Company, 1991

4. Mueller DP, Dolan KD, Yuh WTC.- Imaging case study of the month fibrous dysplasia of the sphenoid sinus. Ann Otol Rhinol Laryngol 1992;101:100-101.

5. Weisman JS, Major USAF, Hepler RS, Vinters HV. Reversible visual loss caused by fibrous dysplasia. Am J of Ophthal 1990;110(3):244-249.

6. Nakagawa K, Takasato Y, Ito Y, Yamada K. Ossifying fibroma involving the paranasal sinuses, orbit, and anterior cranial fossa: case report. Neurosurgery 1995;36(6):1192-5. 